\title{
Confrontation of the Magnetically Arrested Disc Scenario with Observations of FR II Sources
}

\author{
Katarzyna Rusinek* and Marek Sikora \\ Nicolaus Copernicus Astronomical Center, Polish Academy of Sciences, Warsaw, Poland
}

The main aim of our work was to check whether powers of jets in FR II radio galaxies (RGs) and quasars (QSOs) can be reproduced by the Magnetically Arrested Disc (MAD) scenario. Assuming that established in the recent numerical simulations of the MAD scenario the $(H / R)^{2}$ dependence of the jet production efficiency is correct, we demonstrate that in order to reproduce the observed jet powers in FRII sources: (i) accretion discs must be geometrically much thicker than the standard ones; (ii) and/or that the jet production is strongly modulated.

\section{OPEN ACCESS}

Edited by:

Paola Marziani,

Osservatorio Astronomico di Padova

(INAF), Italy

Reviewed by:

Milan S. Dimitrijevic,

Belgrade Astronomical Observatory,

Serbia

Brian Punsly,

International Center for Relativistic

Astrophysics, Italy

*Correspondence:

Katarzyna Rusinek

krusinek@camk.edu.p

Specialty section:

This article was submitted to

Cosmology,

a section of the journal

Frontiers in Astronomy and Space

Sciences

Received: 29 August 2017 Accepted: 27 September 2017

Published: 12 October 2017

Citation:

Rusinek K and Sikora M (2017) Confrontation of the Magnetically

Arrested Disc Scenario with Observations of FR II Sources. Front. Astron. Space Sci. 4:22. doi: 10.3389/fspas.2017.00022
Keywords: active galactic nuclei (AGN), quasars, relativistic jets, accretion discs, magnetically arrested disc (MAD)

\section{INTRODUCTION}

The primary division of Active Galactic Nuclei (AGNs) is based on their radio loudness which is defined as the ratio of radio luminosity (typically $5 \mathrm{GHz}$ ) to the optical luminosity (typically in B band). Minority of AGNs are known to be radio-loud (10\% in case of QSOs, Kellermann et al., 1989) and their radio loudness is sometimes even up to 3-4 orders of magnitude higher than that of radio-quiet AGNs. Moreover, which was found in last years (Rawlings and Saunders, 1991; Punsly, 2007; Fernandes et al., 2011; Sikora et al., 2013), jet powers $P_{j}$ of many radio galaxies reach values comparable to the accretion powers $\dot{M} c^{2}$, where $\dot{M}$ is the mass accretion rate. In order to produce jets with such high efficiencies, $\eta_{j} \equiv P_{j} /\left(\dot{M} c^{2}\right) \simeq 1$, by the Blandford and Znajek (1977) mechanism, the amount of the magnetic flux required to be accumulated on the black hole $(\mathrm{BH})$ is so large that it can only be maintained if it is confined by the ram pressure of the accreting plasma (Sikora and Begelman, 2013). This affects the accretion flow in such a way that the innermost portion of the accretion flow is dynamically dominated by the poloidal magnetic field causing that accretion proceeds via interchange instabilities. This scenario is called Magnetically Arrested Disc (MAD, Narayan et al., 2003; Igumenshchev, 2008; Punsly et al., 2009; Tchekhovskoy et al., 2011; McKinney et al., 2012).

Even though the MAD scenario appears to be an attractive and plausible way to explain the existence of the most powerful jets in radio-loud FR II AGNs (which as described by Fanaroff and Riley, 1974, are characterized by edge-brightened radio structures in contrast to centre-brightened RGs of class I), there are still some open problems. One of them is to establish which parameters truly decide on the MAD occurrence. Assuming that the jet production efficiency depends mostly on the BH spin, van Velzen and Falcke (2013) showed that in fact it is not a dominant parameter for powering jets. Furthermore, since the observed mean efficiency of their FR II quasars sample is much lower than maximal predicted by the MAD model, they concluded that this mechanism does not occur in these sources. On the other hand, in their recent studies, Avara et al. (2016) performed numerical simulations of thin MADs in order to investigate its efficiencies. They found 
that not only $\mathrm{BH}$ spin $a$ but also a geometrical thickness $H / R$ (where $H$ is the disc height and $R$ is the distance from the black hole) contributes to the jet power efficiency as $\eta_{j} \sim a^{2}(H / R)^{2}$. Their results confirm that the MAD scenario can be responsible for explaining powerful jet systems but also point out that at moderate accretion rates discs must be geometrically thicker than the standard theory predicts.

Given the above we checked whether jet powers in FR II radio galaxies and quasars can be reproduced by the MAD scenario. Our studies were thoroughly described in Rusinek et al. (2017). In this paper we briefly outline samples we used and discuss the origins of results we obtained, especially focusing on the importance of geometrical thickness of accretion discs and modulation of jet production.

\section{SAMPLES AND ANALYSIS}

In order to adequately assess the distribution of radio galaxies and quasars in the $P_{j} / L_{d}-\lambda_{\text {Edd }}$ plane (where $L_{d}$ is the bolometric disc luminosity and $\lambda_{\mathrm{Edd}}$ is the Eddington ratio) we have combined four different samples of these sources. We used radio and optical data to calculate necessary values. Including both low- as well as high-redshift objects allowed us to check if cosmological evolution of jet production may have an impact on our final results.

\subsection{Samples}

1. FR II Narrow-Line Radio Galaxies (NLRGs) were extracted from the sample of $z<0.4$ radio galaxies with extended radio structure selected by Sikora et al. (2013). The objects are taken from Cambridge catalogues and matched with the SDSS (Sloan Digital Sky Survey), FIRST (Faint Images of the Radio Sky at $20 \mathrm{~cm}$ ) and NVSS (National Radio Astronomy Observatory (NRAO) Very Large Array (VLA) Sky Survey) catalogues. Due to the available optical data, this sample contains 152 sources;

2. FR II quasar sample was obtained by van Velzen et al. (2015) on the selection of double-lobed radio sources from the FIRST survey catalog, and cross-matching with SDSS quasars. The $\mathrm{BH}$ masses and Eddington ratios, when available, were taken from Shen et al. (2011) thereby reducing the sample from 458 to 414 objects;

3. FR II NLRGs selected in $0.9<z<1.1$ were taken from Fernandes et al. (2011, 2015). The main reason of adding these sources was to verify how much the incompletness of very massive BHs in the local Universe affects the jet production efficiency FR II NLRGs (the first sample). This sample contains 27 objects;

4. Low-redshift Broad-Line Radio Galaxies (BLRGs) and radioloud quasars (RLQs) were used by Sikora et al. (2007) to study radio loudness of these objects. We decided to add this sample (86 sources) to check if the incompletness of SDSS quasars at moderate accretion rates (the second sample) can significantly influence the average value of $P_{j} / L_{d}$ of the FR II quasar sample.

\subsection{Jet Production Efficiency}

For each of mentioned in the previous paragraph samples we calculated the following properties:

- The jet power $P_{j}$ was obtained from the different radio luminosities (from the range of $151 \mathrm{MHz}$ to $5 \mathrm{GHz}$ ) according to the formula based on calorimetry of radio lobes which was proposed by Willott et al. (1999) as

$$
P_{j}\left[\mathrm{erg} \mathrm{s}^{-1}\right]=5.0 \times 10^{22}(f / 10)^{3 / 2}\left(L_{1.4}\left[\mathrm{~W} \mathrm{~Hz}^{-1}\right]\right)^{6 / 7}
$$

where $f$ is the parameter accounting for errors in the model assumptions. Its value (typically between 10 and 20, Blundell and Rawlings, 2000) is established based on comparing jet powers of luminous FR II sources calculated from the model of hotspots Godfrey and Shabala (2013) and the one provided by Willott et al. (1999), and is adpoted as $f=10$;

- The bolometric disc luminosity $L_{d}$ which is related to the line or optical/IR luminosities via respective bolometric corrections. It is used as a proxy of the accretion power $\dot{M} c^{2}=$ $L_{d} / \epsilon_{d}$ where $\epsilon_{d}$ is the disc radiation efficiency depending on $\mathrm{BH}$ spin. In standard disc theory it is assumed to be $\epsilon_{d}=0.1$;

- The Eddington luminosity $L_{\mathrm{Edd}}$ which is necessary to establish the Eddington ratio $\lambda_{\text {Edd }} \equiv L_{d} / L_{\text {Edd }}$ describing the accretion rate, $L_{\mathrm{Edd}} \propto M_{\mathrm{BH}}$ where $M_{\mathrm{BH}}$ is the $\mathrm{BH}$ mass.

We have adopted the $\Lambda$ cold dark matter $(\Lambda \mathrm{CDM})$ cosmology with $H_{0}=70 \mathrm{~km} \mathrm{~s}^{-1}, \Omega_{m}=0.3$ and $\Omega_{\Lambda}=0.7$. Uncertainties of $P_{j} / L_{d}$ and $\lambda_{\text {Edd }}$, calculated as standard deviations of ratios of two independently determined quantities, are not bigger than $0.4 \mathrm{dex}$ for each of the samples. All details about samples and methods used to estimate our calculations are described in Rusinek et al. (2017).

\subsection{Results}

The dependence of $P_{j} / L_{d}$ on the $\lambda_{\text {Edd }}$ for all four samples is presented in Figure 1. The distribution of all objects confirms the trend of the increase of $P_{j} / L_{d}$ with decreasing Eddington ratio (Sikora et al., 2007). All samples are pretty consistent with each other: $z<0.4$ FR II NLRGs and $0.9<z<1.1$ NLRGs samples have similar median of $P_{j} / L_{d}$ at moderate accretion rates (which corresponds to $\lambda_{\text {Edd }}>0.003$ ); FR II quasars and BLRGs+RLQs samples overlap each other.

The horizontal dashed line marks the upper limits for $P_{j} / L_{d}$ predicted by the MAD model for the maximal $\mathrm{BH}$ spin and adopting $\epsilon_{d}=0.1$. The inclined dashed line results from the dependence of the jet production efficiency on $\mathrm{H} / \mathrm{R}$ given as

$$
\eta_{j} \simeq 4 a^{2}\left(1+\frac{0.3 a}{1+2(H / R)^{4}}\right)^{2}(H / R)^{2}
$$

(Avara et al., 2016). For $0.03<\lambda_{\text {Edd }}<1.0$ discs have $H / R \geq 1$ and then such a dependence disappears. The existence of objects in the shaded area, which represents a significant fraction of all studied sources, contradicts with the above predictions and challenges current jet production theories. 


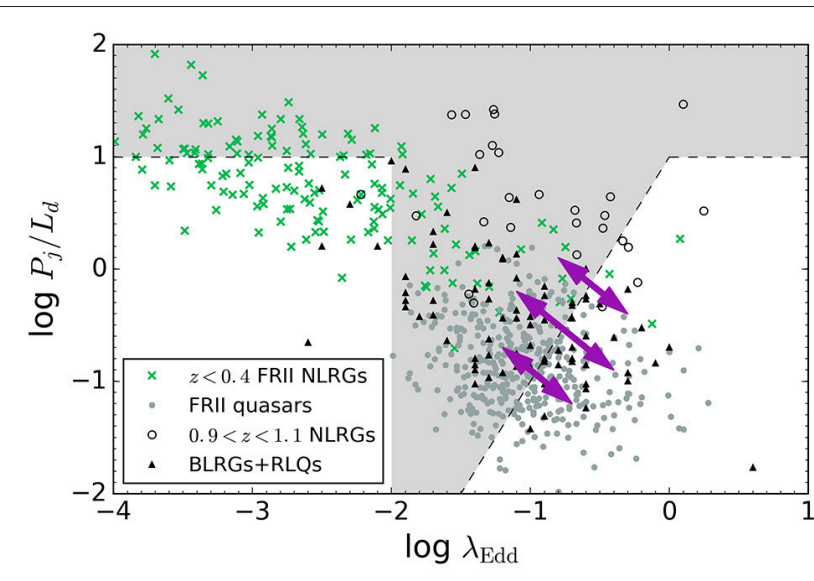

FIGURE 1 | The dependence of $P_{j} / L_{d}$ on the $\lambda_{\text {Edd }}$ for all samples: $z<0.4$ FR II NLRGs as green crosses; FR II quasars as grey dots; $0.9<z<1.1$ NLRGs as empty circles; BLRGs+RLQs as black triangles. The horizontal dashed line corresponds to $P_{j}=\left(\epsilon_{d} / 0.1\right) \dot{M} C^{2}$. The inclined dashed line is derived from the Equation (2) for $a=0.5$ and assuming a correct normalization. The presence of objects in the shaded region of the plot calls into question our underlying assumptions. Purple arrows show how the modulation of jet production affects the $P_{j} / L_{d}-\lambda_{\text {Edd }}$ distribution.

\section{DISCUSSION}

Depending on the Eddington ratio few different explanations for obtained by us jet powers can be proposed. The transition from Radiatively Inefficient, optically thin Accretion Flows (RIAF) to the standard, optically thick accretion discs occurs at $\lambda_{\text {Edd }} \simeq 0.01$ (Best and Heckman, 2012). At lower Eddington ratios the disc radiative efficiency $\epsilon_{d}$ is expected to be much lower than usually assumed 0.1 and this can explain the presence of objects with $P_{j} / L_{d}>10$ in this area. At higher Eddington ratios there are still some sources with $P_{j} / L_{d}>10$. All of them belong to the $0.9<$ $z<1.1$ NLRGs sample for which the bolometric disc luminosity was calculated from the mid-IR data. As it was pointed out by Ogle et al. (2006) this method is not very accurate and therefore discs luminosities of sources taken from Fernandes et al. (2011) may be overestimated.

The presence of objects in the shaded area corresponding to moderate accretion ratios and above the sloping dashed line may indicate that the accretion discs are thicker than the standard ones or/and that the jet production is modulated. However it should be noted here that the extension of the shaded area towards $P_{j} / L_{d} \ll 1$ on the Figure 1 may be inappropriate by noting that the dependence of the jet production efficiency on geometrical thickness of the disk given by Equation (2) can be overestimated resulting from the very approximate treatment of the radiative transfer in Avara et al. (2016) simulations and by not including contribution to the vertical pressure in the MAD zone from toroidal magnetic fields.

\subsection{Thicker Accretion Discs}

Assuming maximally rotating black hole with $a=1$ and producing radiation at a rate $\lambda_{\mathrm{Edd}} \sim 0.01$, the standard accretion disc model (Novikov and Thorne, 1973; Laor and Netzer, 1989) predicts that the maximal value of geometrical thickness of the disc is $H / R \sim 0.4$ which gives the jet production efficiency equal to 0.01 (from the Equation 2). This value is much lower than the median value of FR II quasars sample as well as its upper bound in the $P_{j} / L_{d}-\lambda_{\text {Edd }}$ plot. This discrepancy can be explained if instead of optically thick, geometrically thin standard disc the thicker ones will be considered. These kind of discs have been proposed to avoid gravitational and thermal instabilities in gas and radiation pressure supported discs and they can be formed in presence of strong toroidal magnetic fields (e.g., Begelman and Pringle, 2007; Sądowski, 2016).

Different approach assumes the existence of moderately hot, optically thick and massive layer on top of the relatively colder, accretion disc which stays geometrically thin and optically thick as it was originally developed by Shakura and Sunyaev (1973). Różańska et al. (2015), and Begelman et al. (2015) proved that the model of heavly, viscously driven corona is real if the disc/corona system is stabilized by either strong magnetic fields or vertical outflows. The massive, dense coronas can also arise during the transition from Shakura and Sunyaev (1973) discs to AdvectionDominated Acretion Flows (ADAFs, Abramowicz et al., 1995; Narayan and Yi, 1995) which the transition coincides with the Luminous Hot Accretion Flow (LHAF, Yuan, 2003; Yuan and Narayan, 2014).

\subsection{Modulation of Jet Production}

Another worth considering idea which may explain the visible $P_{j} / L_{d}-\lambda_{\text {Edd }}$ distribution is taking into account the variability of accretion rate. The method we used to establish jet powers is based on the total energy content of the radio lobes. Their lifetime is long (even up to $10^{8}$ years, Komissarov and Gubanov, 1994) so the only possibility of observing variations of jet powers is connected with the hotspot luminosity which may vary on much shorter time-scales. Hotspots however make a small contribution to the total radio luminosity (Mullin et al., 2008) as a result of which jet powers from integrated lobe luminosities are not significantly affected by the variability of the accretion rate. This property may be noticed in the disc luminosity though, which is a direct measure of instantaneous accretion rate. Taken together these findings implicate a modulation of both, the "apparent" jet production efficiency as well as the Eddington ratio in such a way that with decreasing $\lambda_{\text {Edd }}$ the $P_{j} / L_{d}$ increases. This causes the stretching with the slope -1 on the plot which is presented as the set of arrows on the Figure 1. just as the sources are distributed. Variability of accretion rate may be naturally caused by the viscous instabilities in accretion discs (Janiuk et al., 2002; Janiuk and Czerny, 2011).

\section{SUMMARY}

In this proceeding we argue that jets in FR II radio galaxies and quasars accreting at moderate accretion rates are not only powered by MAD scenario but they are also much more powerful than this mechanism predicts. We highlight two possible reasons for this discrepancy. The first one indicates that the geometrical thickness of accretion flows can have much bigger impact on jets formation and evolution than it was originally considered. Here we point out that the future work should concentrate on 
the existence of thicker than the standard accretion discs as well as on systems with massive, hot coronas. The second solution focuses on the variability of accretion rate which can significantly influence observed $P_{j} / L_{d}-\lambda_{\text {Edd }}$ distribution. Since estimated by us jet powers are based on the calorimetry on the radio lobes and thereby they are averaged over the lifetime of the source, an important issue to resolve for future studies is to check if using different methods for calculating jet powers such as X-ray cavities (Cavagnolo et al., 2010; Nemmen and Tchekhovskoy, 2015) or model of hotspots (Godfrey and Shabala, 2013) such a trend of decreasing $\lambda_{\text {Edd }}$ with increasing $P_{j} / L_{d}$ will be still visible.

\section{AUTHOR CONTRIBUTIONS}

Both authors made substantial, direct and intellectual contribution to the work. KR collected and analyzed presented

\section{REFERENCES}

Abramowicz, M. A., Chen, X., Kato, S., Lasota, J.-P., and Regev, O. (1995). Thermal equilibria of accretion disks. Astrophys. J. 438, L37-L39. doi: 10.1086/187709

Avara, M. J., McKinney, J. C., and Reynolds, C. S. (2016). Efficiency of thin magnetically arrested discs around black holes. Mon. Not. R. Astron. Soc. 462:636. doi:10.1093/mnras/stw1643

Begelman, M. C., and Pringle, J. E. (2007). Accretion discs with strong toroidal magnetic fields. Mon. Not. R. Astron. Soc. 375, 1070-1076. doi: 10.1111/j.1365-2966.2006.11372.x

Begelman, M. C., Armitage, P. J., and Reynolds, C. S. (2015). Accretion disk dynamo as the trigger for X-ray binary state transitions. Astrophys. J. 809:118. doi: $10.1088 / 0004-637 X / 809 / 2 / 118$

Best, P. N., and Heckman, T. M. (2012). On the fundamental dichotomy in the local radio-AGN population: accretion, evolution and host galaxy properties. Mon. Not. R. Astron. Soc. 421, 1569-1582. doi: 10.1111/j.1365-2966.2012.20414.x

Blandford, R. D., and Znajek, R. L. (1977). Electromagnetic extraction of energy from Kerr black holes. Mon. Not. R. Astron. Soc. 179, 433-456. doi: 10.1093/mnras/179.3.433

Blundell, K. M., and Rawlings, S. (2000). The spectra and energies of classical double radio lobes. Astron. J. 119, 1111-1122. doi: 10.1086/301254

Cavagnolo, K. W., McNamara, B. R., Nulsen, P. E. J., Carilli, C. L., Jones, C., and Birzan, L. (2010). A relationship between AGN jet power and radio power. Astrophys. J. 720, 1066-1072. doi: 10.1088/0004-637X/720/2/1066

Fanaroff, B. L., and Riley, J. M. (1974). The morphology of extragalactic radio sources of high and low luminosity. Mon. Not. R. Astron. Soc. 167:31P. doi: 10.1093/mnras/167.1.31P

Fernandes, C. A. C., Jarvis, M. J., Rawlings, S., Martinez-Sansigre, A., Hatziminaoglou, E., Lacy, M., et al. (2011). Evidence for a maximum jet efficiency for the most powerful radio galaxies. Mon. Not. R. Astron. Soc. 411, 1909-1916. doi: 10.1111/j.1365-2966.2010.17820.x

Fernandes, C. A. C., Jarvis, M. J., Martínez-Sansigre, A., Rawlings, S., Afonso, J., Hardcastle, M. J., et al. (2015). Black hole masses, accretion rates and hot- and cold-mode accretion in radio galaxies at $\mathrm{z} \sim 1$. Mon. Not. R. Astron. Soc. 447, 1184-1203. doi:10.1093/mnras/stu2517

Godfrey, L. E. H., and Shabala, S. S. (2013). AGN jet kinetic power and the energy budget of radio galaxy lobes. Astrophys. J. 767:12. doi: 10.1088/0004-637X/767/1/12

Igumenshchev, I. V. (2008). Magnetically arrested disks and the origin of poynting jets: a numerical study. Astrophys. J. 677, 317-326. doi: 10.1086/529025

Janiuk, A., and Czerny, B. (2011). On different types of instabilities in black hole accretion discs: implications for X-ray binaries and active galactic nuclei. Mon. Not. R. Astron. Soc. 414, 2186-2194. doi: 10.1111/j.1365-2966.2011.18544.x

Janiuk, A., Czerny, B., and Siemiginowska, A. (2002). Radiation pressure instability driven variability in the accreting black holes. Astrophys. J. 576, 908-922. doi:10.1086/341804 data. MS formulated the original problem, provided direction and guidance. Both authors contributed to the discussion of the obtained results.

\section{FUNDING}

The research leading to these results has received funding from the Polish National Science Centre grant 2016/21/B/ST9/01620.

\section{ACKNOWLEDGMENTS}

We are particularly grateful to Dorota Kozieł-Wierzbowska and Leith Godfrey for their significant contribution in the analysis of the results and a crucial discussion on their possible explanations. We also thank Greg Madejski for helpful comments.

Kellermann, K. I., Sramek, R., Schmidt, M., Shaffer, D. B., and Green, R. (1989). VLA observations of objects in the Palomar Bright Quasar Survey. Astron. J. 98, 1195-1207. doi: 10.1086/115207

Komissarov, S. S., and Gubanov, A. G. (1994). Relic radio galaxies: evolution of synchrotron spectrum. Astron. Astrophys. 285, 27-43

Laor, A., and Netzer, H. (1989). Massive thin accretion discs. I - Calculated spectra. Mon. Not. R. Astron. Soc. 238, 897-916. doi: 10.1093/mnras/238.3.897

McKinney, J. C., Tchekhovskoy, A., and Blandford, R. D. (2012). General relativistic magnetohydrodynamic simulations of magnetically choked accretion flows around black holes. Mon. Not. R. Astron. Soc. 423, 3083-3117. doi: 10.1111/j.1365-2966.2012.21074.x

Mullin, L. M., Riley, J. M., and Hardcastle, M. J. (2008). Observed properties of FRII quasars and radio galaxies at $\mathrm{z}<1.0$. Mon. Not. R. Astron. Soc. 390, 595-621. doi: $10.1111 /$ j.1365-2966.2008.13534.x

Narayan, R., and Yi, I. (1995). Advection-dominated accretion: self-similarity and bipolar outflows. Astrophys. J. 444, 231-243. doi: 10.1086/175599

Narayan, R., Igumenshchev, I. V., and Abramowicz, M. A. (2003). Magnetically arrested disk: an energetically efficient accretion flow. Publ. Astron. Soc. Japan 55, L69-L72. doi: 10.1093/pasj/55.6.L69

Nemmen, R. S., and Tchekhovskoy, A. (2015). On the efficiency of jet production in radio galaxies. Mon. Not. R. Astron. Soc. 449, 316-327. doi: $10.1093 / \mathrm{mnras} / \mathrm{stv} 260$

Novikov, I. D., and Thorne, K. S. (1973). “Astrophysics of black holes," in Black Holes (Les Astres Occlus), eds C. Dewitt and B. S. Dewitt (New York, NY: Gordon and Breach Science Publishers), 343-450.

Ogle, P., Whysong, D., and Antonucci, R. (2006). Spitzer reveals hidden quasar nuclei in some powerful FR II radio galaxies. Astrophys. J. 647:161. doi: $10.1086 / 505337$

Punsly, B. (2007). Kinetically dominated FRII radio sources. Mon. Not. R. Astron. Soc. 374, L10-L14. doi: 10.1111/j.1745-3933.2006.00254.x

Punsly, B., Igumenshchev, I. V., and Hirose, S. (2009). Three-dimensional simulations of vertical magnetic flux in the immediate vicinity of black holes. Astrophys. J. 704, 1065-1085. doi: 10.1088/0004-637X/704/2/1065

Rawlings, S., and Saunders, R. (1991). Evidence for a common centralengine mechanism in all extragalactic radio sources. Nature 349, 138-140. doi: $10.1038 / 349138 \mathrm{a} 0$

Różańska, A., Malzac, J., Belmont, R., Czerny, B., and Petrucci, P.-O. (2015). Warm and optically thick dissipative coronae above accretion disks. Astron. Astrophys. 580:A77. doi: 10.1051/0004-6361/201526288

Rusinek, K., Sikora, M., Kozieł-Wierzbowska, D., and Godfrey, L. (2017). On the efficiency of jet production in FR II radio galaxies and quasars. Mon. Not. R. Astron. Soc. 466, 2294-2301. doi: 10.1093/mnras/stw3330

Sądowski, A. (2016). Thin accretion discs are stabilized by a strong magnetic field. Mon. Not. R. Astron. Soc. 459, 4397-4407. doi: 10.1093/mnras/stw913

Shakura, N. I., and Sunyaev, R. A. (1973). Black holes in binary systems. Observational appearance. Astron. Astrophys. 24, 337-355 
Shen, Y., Richards, G. T., Strauss, M. A., Hall, P. B., Schneider, D. P., Snedden, S., et al. (2011). A catalog of quasar properties from sloan digital sky survey data release 7. Astrophys. J. 194:45. doi: 10.1088/0067-0049/194/2/45

Sikora, M., and Begelman, M. C. (2013). Magnetic flux paradigm for radio loudness of active galactic nuclei. Astrophys. J. 764:L24. doi: 10.1088/2041-8205/764/2/L24

Sikora, M., Stawarz, Ł., and Lasota, J.-P. (2007). Radio loudness of active galactic nuclei: observational facts and theoretical implications. Astrophys. J. 658, 815-828. doi: 10.1086/511972

Sikora, M., Stasińska, G., Kozieł-Wierzbowska, D., Madejski, G. M., and Asari, N. V. (2013). Constraining jet production scenarios by studies of narrow-line radio galaxies. Astrophys. J. 765:62. doi: 10.1088/0004-637X/765/1/62

Tchekhovskoy, A., Narayan, R., and McKinney, J. C. (2011). Efficient generation of jets from magnetically arrested accretion on a rapidly spinning black hole. Mon. Not. R. Astron. Soc. 418, L79-L83. doi: 10.1111/j.1745-3933.2011.01147.x

Yuan, F. (2003). Luminous hot accretion flows: thermal equilibrium curve and thermal stability. Astrophys. J. 594, L99-L102. doi: 10.1086/378666

Yuan, F., and Narayan, R. (2014). Hot accretion flows around black holes. Annu. Rev. Astron. Astrophys. 52, 529-588. doi: 10.1146/annurev-astro-082812141003 van Velzen, S., and Falcke, H. (2013). The contribution of spin to jet-disk coupling in black holes. Astron. Astrophys. 557:L7. doi: 10.1051/0004-6361/201322127

van Velzen, S., Falcke, H., and Körding, E. (2015). Nature and evolution of powerful radio galaxies at $\mathrm{z} \sim 1$ and their link with the quasar luminosity function. Mon. Not. R. Astron. Soc. 446, 2985-3001. doi: $10.1093 / \mathrm{mnras} / \mathrm{stu} 2213$

Willott, C. J., Rawlings, S., Blundell, K. M., and Lacy, M. (1999). The emission lineradio correlation for radio sources using the 7C Redshift Survey. Mon. Not. $R$. Astron. Soc. 309, 1017-1033. doi: 10.1046/j.1365-8711.1999.02907.x

Conflict of Interest Statement: The authors declare that the research was conducted in the absence of any commercial or financial relationships that could be construed as a potential conflict of interest.

Copyright (๑) 2017 Rusinek and Sikora. This is an open-access article distributed under the terms of the Creative Commons Attribution License (CC BY). The use, distribution or reproduction in other forums is permitted, provided the original author(s) or licensor are credited and that the original publication in this journal is cited, in accordance with accepted academic practice. No use, distribution or reproduction is permitted which does not comply with these terms. 